\title{
Author Index for Volume 108
}

(Bill) Musk, AW 1879

Abbadessa, G 21

Abbas, M 131

Abbas, R 1985

Abdallah, Y 2448

Abdel-Rahim, M 973

Abe, K 1415

Abe, Y 1488

Abel, GA 686, 1550, 2187

Abel, PD 2192

Åberg, O 342

Abildgaard, MO 420

Abnet, CC 1378

Aboagye, EO 342

Abol- Enein, H 973

Abraham, J 866

Absenger, G 1677

Acres, B 2045

Adamopoulos, C 2142

Adams, CE 139

Adams, E 1602

Addington-Hall, J 1942

Agaimy, A 2164

Agelaki, S 549

Agostini, M 278

Agrawal, S 1616

Agudo, A 708

Aguggini, S 1587

Ahmad, AS 1215

Ahmad, I 149

Ahrensberg, JM 1280

Aigelsreiter, A 1830

Akahira, J 1415

Akanuma, N 644

Akiba, S 229

Akintayo, A 762

Akiyama, F 1921

Akiyama, Y 932

Akutsu, Y 644

Alabiso, O 1743

Al-Attar, A 2097

Al-Batran, S-E 265, 519

Albelda, SM 1288, 1306

Albertsen, PC 1534

Albino, V 1566

Alcaraz, A 2565

Aldridge, J 1273

Alentorn, A 2178

Ali, HR 602

Allegrini, S 1743

Allemani, C 1195

Allen, D 2045

Allen, N 708

Allevi, G 1587

Almadori, G 1157

Aloj, L 1566

Alsadius, D 1964

Alsop, DC 319

Altevogt, P 1449

Altman, DG 2205

Álvarez-Ossorio, JL 2565

Alvarez-Rodriguez, FJ 2334

Alvarez-Vega, MA 2005

Amador-Sanchez, R 2334

Amiot, M 1801

Amirian, ES 1173

Amundson, SA 91, 1106

Andäng, M 2191

Andersen, L 715

Andersen, TV 2346

Anderson, $\mathrm{H} 812$

Anderson, KE 727

Anderson, LA 1178

Andreis, D 1587

Andre, N 2435

Anido, U 2565
Antczak, A 461, 2601

Antolín, I 2005

Antona, J 1743

Antoniotti, C 2549

Aoyma, T 1495

Apostolaki, S 549

Arai, T 1495

Aravani, A 681

Arden-Close, E 1004

Ardill, J 301

Ardizzoni, A 1695

Arellano-Galindo, J 2334

Arem, H 1168

Arenas Hernandez, M 2505

Argüelles Suárez, MV 708

Arita, T 361, 1324, 1822

Armeanu-Ebinger, S 334

Armesilla, AL 994

Armstrong, DK 1231

Armstrong, G 2178

Armstrong, J 1634

Arnesson, L-G 812

Asaka, M 1488

Aschebrook-Kilfoy, B 205

Ashcroft, GP 1883

Ashdown-Barr, L 908

Ash, S 1092

Ashworth, A 983

Askenasy, N 1092

Asselain, B 1807

Assinder, SJ 409

Atanasov, AG 570

Atkins, MB 319

Atmaca, A 265

Austin, J 908

Austin, RC 327

Autorino, R 1157

Avgeris, M 2573

Awada, A 1641

Awasthi, S 557

Aydin, D 2346

Azzoni, C 1695

Babur, M 1704

Badran, B 1641

Bae, JM 1978

Baglietto, L 743

Bago-Horvath, Z 1100

Bahamon, B 319

Baidoo, KE 2013

Baiter, M 2164

Baker, L 873

Baldini, E 1695

Balic, M 1408

Ballester, M 1267

Balta, S 2625

Balzarini, L 58

Banat, A-G 265

Banerjee, S 2264

Bangma, CH 533, 1971

Bang, Y-J 1978

Banjevic, D 542

Banks, M 1440

Banks, RE 1133

Barbie, T 1480

Barbouni, A 199

Barcenas, CH 285

Bar, K 461

Barker, CE 1634

Barker, EM 450

Barner, M 469

Barnes, DR 2610

Baron, JA 1757

Barone, C 115

Barranger, E 285

Barrett-Lee, P 1195
Barricarte, A 708

Barrios, CH 2470

Barron, C 2021

Barrowdale, D 2610

Barry, M 1211

Barton Grossman, H 2372

Bartsch, R 1100, 2259

Bashyam, MD 2056

Bassi, F 1593

Basso, M 115

Basta, N 2339

Bastian, PJ 973

Batra, SK 1079, 2590

Bauer, J 2164

Bauer, S 564

Baum, M 820, 2198

Baxter, RC 351

Bayne, AM 2455

Bazzola, L 1587

Bazzoli, F 1750

Beare, S 1252

Beattie, CW 1061, 2495

Beatty, LK 327

Beeken, RJ 831

Beekman, ATF 2628

Beesley, J 2610

Behrens, G 798

Behringer, K 49

Bekker-Mendez, VC 2334

Belcher, J 721

Beldiman, C 2304

Bellet, D 1720

Bellmunt, J 311, 2565

Bellò, M 527

Benítez, J 1732

Bennett, H 2250

Beral, V 2390

Berger, J 887

Bergh, J 1195

Berkers, J 887

Bernáldez-Ríos, R 2334

Berndt, S 1378

Berney, DM 271, 149, 2582

Bernhard, H 265

Bernstein, L 727

Berruti, A 1587

Bertelli, G 2250

Bertolini, F 1387

Bertorelle, R 278

Bertossi, M 58

Bettermann, K 1830

Beuselinck, B 887

Bevan, CL 1925

Bevan, P 766

Bhat, AB 1552

Bhat, GA 1552

Bhatt, RS 319

Bhattacharjee, S 1378

Bhattacharyya, H 1571

Bianchi, P 1750

Bianco, R 1616

Bianconi, M 1126

Biasini, L 278

Bièche, I 1720

Bieche, I 1807

Bigot, P 887

Birrer, MJ 1231

Bishop, DT 1502

Bittoni, A 1126

Bizzarri, AR 2495

Bjartell, A 708, 1668

Bjørge, T 1525

Björkholm, M 2272

Black, A 1178

Black, RJ 1883

Blackhall, FH 1704

Blancafort, P 881

Blick, C 1133

Blixt, O 2045

Bloigu, R 1917

Blows, FM 602

Bobe, G 1168

Bodmer, WF 2106

Boeck, S 469, 766, 1684

Boeing, $\mathrm{H} 708$

Boenink, AD 2628

Boer, IGJ 587

Boer, JC 587

Boffetta, P 222, 1391, 1552

Boland, CR 1750

Boldorini, RL 621, 1743

Bolea-Murga, V 2334

Bolton, E 1213

Boman, K 2321

Bomhof-Roordink, H 2628

Bonanni, B 1593

Bonardi, S 1587

Bondarenko, I 1985

Bondy, M 2178

Boni, C 1695

Boni, L 1695

Boniface, D 292

Bonifacio, C 58

Bonnaud, S 1801

Borchmann, P 49

Bordi, C 1695

Bordi, P 1695

Bordignon, C 58

Borjigin, N 653

Borkowski, A 461, 2601

Borkowski, T 461, 2601

Borre, M 420

Borrego, S 1732

Bortesi, B 1695

Boshuizen, H 708

Bosman, FT 676

Bosserhoff, AK 1460

Botelho, F 2354

Botteri, E 1593

Bottini, A 1587

Bottomley, D 1495

Boudou-Rouquette, P 1034

Bougnoux, P 1771

Bourke, L 9, 2194

Bourne, T 2448

Bouvier, AM 775

Bouvier, V 775

Boviatsis, E 2142

Bowden, S 866

Bowen, RL 2195

Boyages, J 1195

Boyle, FM 351

Braamse, AM 2628

Bracarda, S 311

Braga, M 848 
Brinkworth, E 2250

Brinton, LA 727, 1378

Britton, P 2442

Broderick, P 2178

Brodie, H 1936

Brooks, L 1316

Brown, S 994

Brownbill, PA 2455

Broza, YY 941

Brueck, P 265

Brueckner, A 311

Bruix, J 21

Brundel, DHS 1071

Bryan, RT 1854

Bryant, JL 1704

Bryniarski, P 461

Buchanan, DD 1757

Buchán-Durán, EP 2334

Buck, K 188

Buecher, B 1012

Bueno-de-Mesquita, HB 708

Bueti, D 2045

Buffart, TE 1495

Bui, S 1332

Buisine, M-P 2079

Bukowski, R 2470

Bulkescher, J 973

Bultz, BD 738, 2631

Bunch, KJ 1907, 2455

Bunyan, DJ 438

Burattini, L 1126

Burchell, JM 2045

Burdette, L 1378

Burford, B 2045

Burger, DM 1071

Burgos-Morón, E 2189

Burnell, M 107

Burroughs, AK 1252

Buskens, C 2106

Bussolati, B 2525

Bussu, F 1157

Butler, J 1195

Byrski, T 461

Caca, K 265

Cadman, L 908

Cafferty, FH 2192

Cai, Q 380

Cai, W 2542

Calaf, GM 91, 1106

Caldas, C 866, 602, 2442

Calderero, V 2565

Calderón-Montaño, JM 2189

Calegari, MA 115

Caley, MP 163

Calster, BV 2448

Camacho, LH 21

Camacho, N 2582

Cameron, DA 2205

Campanini, N 1695

Cannistraro, S 2495

Caparello, C 2549

Caplin, ME 1838

Caporaso, NE 1218

Cappelletti, MR 1587

Capper, D 924

Cappuzzo, F 668

Caracò, C 1566

Carbajo-Pescador, S 442

Cárdenas-Cardos, R 2334

Carles, J 2565

Carlson, LE 738, 2631

Caron, O 1012

Carre, M 2435

Carter, CR 997

Carter, R 613

Carus, A 2116

Carvalho, B 1495

Casadevall, A 859

Casadio, C 621

Cascinu, S 1126

Cassano, A 115

Cassoni, P 527
Castillo, AD 1593

Castro, C 2354

Catalano, PJ 784

Catley, L 1624

Catt, S 2264

Catto, JWF 1534

Cazorla, A 1732

Cebotaru, C 1027

Ceccarelli, C 1750

Ceder, JA 1668

Ceder, Y 1668

Cella, D 1571

Cenci, T 115

Chai, Y 91, 1106

Chambers, PA 1495

Champ, R 866

Chan, A 1052

Chan, AT 1891

Chandra, D 2281

Chang, C-Y 72

Chang, GJ 1508

Chang, H-M 1245

Chang, Y 2623

Chang-Claude, J 188

Channathodiyil, P 994

Chanock, SJ 1378

Chaouachi, K 1389

Chapman, EJ 1368

Chapman, JW 784

Chatterjee, N 1378

Chatziandreou, I 2142

Chaudhuri, G 848

Cheetham, SW 2419

Chen, C 1571

Chen, C-W 791

Chen, EX 1298

Chen, G 1238

Chen, H 2299

Chen, H-H 2241

Chen, HX 826

Chen, I 1393

Chen, J 1143

Chen, M 2542

Chen, P-C 1778

Chen, W 1470

Chen, X 2610

Chen, Z 409

Chen, Z 762

Chen, Z-d 941

Chen, Z-H 1470

Cheng, A-L 72

Cheng, G 1288

Cheng, KK 1854

Cheng, T-L 791

Chereau, E 1267

Cheung, K-F 2557

Cheung, KL 1042

Chiba, T 395

Chico, TJA 9

Chie, EK 1425

Chieco, P 1750

Chilcott, J 1211

Chin, X 1388

Ching, L-M 1306

Chirlaque, M-D 708

Cho, DH 1862

Cho, N-Y 1978

Choi, EY 1862

Choi, HY 387

Choi, Y 387

Choueiri, TK 2478

Chow, LW-C 1985

Chow, W-H 1378

Christiansen, P 1195

Christov, K 1061, 2495

Chromecki, TF 901

Chua, J 1388

Chua, TC 1432

Chung, L 351

Ciccarino, P 2178

Cilli, M 2525

Ciuleanu, T 1027

Cizkova, M 1807
Claes, B 887

Clark, J 2582

Clarke, HJ 1340

Clausen, H 2045

Clavel, J 1899

Clemens, MR 469

Clifford, GM 240

Cloots, RHE 676

Coffey, RJ 1765

Coleman, MP 1195

Coleman, N 450

Collette, EH 2628

Colombo, N 1021

Colomer, R 1298

Comber, H 1211

Comet-Batlle, J 2565

Connor, T 1624

Constela, M 2565

Contreras, JA 2565

Cook, LS 727

Cooke, R 959, 2399

Coombes, EG 775

Cooper, C 149

Cooper, CS 2582

Corcoran, NM 1388

Cornet, I 240

Corrigan, A 2505

Cortés, J 1052

Corver, WE 873

Costas, L 742

Costello, AJ 1388

Costello, E 1846

Cotton, SJ 721

Cottu, P 1720

Couchy, G 887

Coutant, C 285

Cox, T 1846

Cox, TF 1942

Coyle, B 2130

Coyle, VM 1027

Coyne, JC 736

Cozen, W 1163

Cozzolino, I 1616

Cremolini, C 2549

Crispo, A 222

Crolla, JA 959

Cross, AJ 205

Crouch, S 1213

Cunningham, D 493, 983, 2399

Cuthbertson, DJ 1440

Cutz, J-C 2021

Cuzick, J 149, 271, 908, 2582

Cvek, B 993

Cybulski, C 461, 2601

Dadachova, E 859

Dagnæs-Hansen, F 2291

Dahiya, R 1659, 2070

Dahlin, AM 2153

Daibata, M 629, 2624

Dal Moro, F 2415

Dalton, LE 1340

Damery, S 1149

Damiano, V 1616

D’Angelo, L 1750

Dangles-Marie, V 1720

Daniel, CR 205

Daniele, G 1566

Dar, NA 1391, 1552

Daraï, E 1267

Darby, SC 179

Darcy, KM 613, 1231

Darling, JL 994

Darmanin, J 1402

Dart, DA 1925

Das, K 1495

Das, S 2590

Das Gupta, TK 1061

Datta, K 2590

Daugaard, G 1994

David, L 2045

Davies, N 1252

Davis, MPA 450

Dawnay, A 107, 2045

Dawood, S 745

Dawsey, SM 1378

Dawson, H 2088

Dawson, S-J 602

Dayal, JHS 873

Dean, EJ 1704

deBekker-Grob, EW 533

Dębniak, T 461, 2601

de Bock, GH 1579

DeCensi, A 1593

de Cremoux, P 1807

de Diego Flores-Chapa, J 2334

De Dosso, S 668

De Faveri, LE 1368

de Haas, CJC 587

de Haas, SL 1052

Dejardin, O 775

Dejong, CHC 676

Dekker, J 2628

de Klerk, N 1879

de Koning, HJ 1971

De Laere, B 1358

del Alba, AG 2565

de la Piedra, C 2565

de la Taille, A 887

Delattre, J-Y 2178

del Carmen Rodriguez-Zepeda, $M$ 2334

deLeeuw, RJ 155

Delgado, D 2565

DellaValle, CT 205

Delmar, PR 1052

Del Mistro, A 240

Delogu, G 1157

DeLoia, JA 1231

Delongchamps, NB 1034

Delpech, Y 285

Del Prete, M 1126

Delrio, P 1566

de Lutio di Castelguidone, E 1566

deMartin, R 564 
Doki, Y 1119

Dolbeault, S 1012

Dolecek, TA 2367

Dolznig, H 564, 570

Domagała, P 461, 2601

Domanska, UM 587

Domingo, S 1732

Dommett, RM 2329

Dong, Q 409

Dong, Y 2557

Donkers, B 533

Donnan, PT 1515

Donnelly, C 292

Donskov, F 2116

Dorantes-Acosta, EM 2334

Dorrius, MD 1579

Douillard, J-Y 493

Downing, A 681

Doyle, LA 826

D'Amelio, P 2525

D'Amico, L 2525

Draisma, G 1971

Draper, GJ 2455

Driessen, A 676

Drought, A 2448

Duan, Z 213

Dubernard, G 1267

Dubrowinskaja, N 131

Dubsky, P 1408, 2259

Duivenvoorden, WC 327

Dujaric, M-E 1807

Duncan, G 1298

Dunn, J 866

Durrani, S 2250

Durrant, L 2097

Durrant, S 2097

Dutcher, J 311

Earl, HM 866

Easton, D 2202

Eberhard, J 2321

Eberhard, K 901

Ebert, MP 766

Edin, S 2153

Edsjö, A 1668

Edwards, J 139

Efstathiou, JA 1225

Egan, KJ 64

Egg, R 469

Eggen, T 2346

Eichenauer, DA 49

Eicher, C 334

Einstein, MH 1021, 2281

Eisele, L 2178

Eiser, C 1004

Eisner, F 1830

Eklöf, V 2153

Elashoff, DA 2033

Elder, EE 351

El-Habr, EA 2142

El Hajj, P 1641

Ellerkamp, V 334

Ellershaw, JE 1942

Elliott, K 163

Elliott, R 983

Elliott, V 1846

Ellis, IO 1042

Elliston, CD 91

El-Omar, EM 2537

El-Rayes, B 762

El-Serag, HB 213

El'skaya, A 2304

Emdin, S 812

Enciso-Mora, V 2178

Engeland, A 1525

Engert, A 49

Engholm, G 1195

English, DR 743

Enokida, H 1648

Enright, AJ 450

Epstein, E 2448

Escande, F 2079

Escudier, B 311, 1571, 2470
Eskandarpour, M 964

Esserman, L 2200

Essink-Bot, M-L 533

Esteban, E 2565

Esteva, FJ 285

Eto, K 1488

Evans, TRJ 997

Fabbro, M 1771

Fairbanks, L 2505

Fajac, A 1012

Fajardo-Gutiérrez, A 2334

Falcone, A 2549

Falk, SJ 2399

Fallowfield, L 1402, 2264

Faloppi, L 1126

Fan, S 1470

Fang, X 380

Fang, Y 1470

Fang, YJ 1238

Fankhauser, M 1388

Fanucchi, MP 762

Faratian, D 2039

Farewell, D 1402

Farewell, V 1402

Favier, L 1771

Fear, NT 1907

Feeney, A 2464

Feenstra, T 1579

Felix, AS 727

Feltbower, RG 1223, 1273

Feng, H 2623

Fentiman, I 2045

Fenwick, N 866

Ferguson, B 1213

Fernández, JM 2565

Fernando, I 2464

Ferracini, R 2525

Ferrari, P 708

Fesl, C 1408

Feychting, M 2346

Fielding, R 1922

Filipits, M 1100

Finan, PJ 681, 1502

Fini, L 1750

Finotto, R 58

FischervonWeikersthal, L 469

Fisher, G 149, 271, 2582

Fisher, R 479

Fitzal, F 1408

Flahault, C 1012

Flanagan, AM 964

Flechtner, H-H 49

Fleming, J 149

Fleming, T 1480

Flesch-Janys, D 188

Floquet, A 1771

Flores-Lujano, J 2334

Flores-Villegas, LV 2334

Fly, K 2470

Fogel, M 1449

Fong Lin, M 2590

Fonseca, A-V 163

Fontani, L 2525

Fontanini, G 1695

Fontes, F 2354

Fora, G 527

Forbes, LJL 292

Ford, J 2045

Foreman, J 2442

Forman, D 775

Formisano, L 1616

Fornander, T 1195

Foroni, C 1587

Fotopoulou, C 32

Fountzilas, G 668

Fourkala, E-O 107, 2045

Fox, SB 1587

Fragkou, P 2142

Fragoulis, EG 2573

Franceschi, S 222, 240

Franke, A 2178

Fraser, LK 1273
Frattini, M 668, 1743

Frederiksen, K 234

Freedman, ND 1378

Freyer, G 1810

Freyman, A 1985

Fricker, SP 1634

Fridlender, ZG 1288

Friedenreich, CM 727

Friend, L 301

Friess, H 1846

Friis, S 715, 1189

Friso, ML 278

Fritschi, L 1879

Fu, S 826

Fuchs, CS 1891

Fuchs, J 334

Fuchs, M 49

Fueyo, J 2005

Fujisawa, T 744

Fujita, J 1119

Fujita, K 653

Fujita, M 1348

Fujiwara, H 1324, 1822

Fujiwara-Okada, Y 836

Fujiwara, S 170, 1119

Fujiwara, T 836

Fukui, H 395

Fukuoka, M 1488

Fukushi, J 836

Funel, N 2549

Furuse, J 2063

Furuyama, N 2516

Fushimi, K 1817

Fyfe, N 2537

Fyffe, G 139

Gagnon, B 1790

Gail, MH 1163

Gaist, D 715, 1189

Galibert, M-D 1641

Galle, PR 442

Galli, J 1157

Gallino, D 327

Gallus, R 1157

Galsky, M 2478

Gammerman, A 2045

Ganju, V 771

Gao, C 2299

Gao, F 1480

Gapstur, SM 727

Garcia, M 1750

García, MJ 1732

García-Bueno, JM 1732

Garcia-Closas, M 1378

García-Donas, J 1732

García-Escudero, A 2565

García-Rodríguez, LA 1189

Garibaldi, J 1042

Garmo, H 812

Gasser, M 1432

Gaudet, MM 727

Gauler, TC 469

Gauthier-Villars, M 1012

Gazdar, AF 744

Gebauer, K 131

Gebski, VJ 771

Gehrig, PA 1021

Geissler, M 469

Gelardi, T 1616

Generali, D 1587

Gentry-Maharaj, A 107, 2045

Georgila, C 199

Georgoulias, V 549, 2426

Gerger, A 901, 1677, 2627

Germer, CT 1432

Gerner, EW 512

Geva, R 668

Ghaderi, S 1525

Ghaem-Maghami, S 2448

Ghandhi, SA 91

Ghanem, G 1641

Gheit, T 240

Ghoneim, MA 973

Ghosh, A 557

Giampieri, R 1126

Giannos, A 1100

Giardini, R 1587

Giessrigl, B 564, 570

Gifford, AJ 2435

Gigi, V 1092

Gilbert, TM 1440

Gilbo, N 527

Giles, GG 743

Gillanders, WE 1480

Gillmore, R 1252

Gilson, D 2399

Giordano, P 1566

Giovannucci, EL 183, 1891

Giroux, J 1034

Giuliano, AE 2033

Gjerstorff, ML 1195

Glaser, A 14, 1223

Glehr, M 1677

Gliniewicz, B 461

Gnanapragasam, VJ 597

Gnant, M 1408, 2259

Godio, L 2525

Goedert, JJ 1163

Gojis, O 2039

Gołab, A 461, 2601

Golbeck, $S 90$

Goldacre, MJ 193

Goldberg, GL 859

Goldberg, SN 319

Goldbohm, RA 727

Goldstein, AM 1378

Goldwasser, F 1034

Golfier, F 1267

Gomes, S 2354

Gomez-Bougie, P 180

Gómez-Caamano, A 2565

Gomez-Manzano, C 2005

Gomez-Navarro, J 1998

Gómez-Veiga, F 2565

González-Enguita, C 2565

González-Gallego, J 442

González-Gragera, B 2565 
Grunfeld, E 14

Grusch, M 564, 570

Guerrieri-Gonzaga, A 1593

Gu, F 1378

Gu, K-s 941

Guinebretière, J-M 1720

Güner, T 265

Gunter, MJR 708

Günther Derigs, H 265

Guo, H 99

Guo, K 1306

Gupta, N 2056

Gupta, S 1985

Gupta, TKD 2495

Gussago, F 1587

Guthrie, G 1915

Guyot-Goubin, A 1899

Ha, YJ 1862

Ha, SW 1425

Haas, M 1684

Habra, MA 826

Hackshaw, A 2464

Haferkamp, A 973

Haflidadóttir, BS 1668

Hager, H 2116

Hagman, Z 1668

Hahn, S 39

Haick, H 941

Hakulinen, T 699

Halai, D 964

Halbsguth, TV 49

Haldrup, C 420

Hale, L 213

Halil Sahin, I 740

Hall, M 250

Hall, P 2202

Hallas, J 715, 1189

Hall-Craggs, MA 2464

Hallett, R 2045

Halonen, P 82

Haluska, F 1021

Hamada, J-i 2516

Hamburger, AW 557

Hamdy, FC 748

Hamilton, AS 1163

Hamilton, W 25, 2329

Hammond, M 2464

Han, SS 1378

Han, S-W 1425, 1978

Hanari, N 644

Hanazawa, T 1648

Hancock, BW 2399

Hankinson, SE 183

Hanning, JE 450

Hansen, RP 1280

Hantusch, B 564

Hao, C 744

Harari, D 1936

Harel-Bellan, A 2304

Harmenberg, U 1541

Harper, P 2505

Harrington, KJ 735

Harris, AL 1133, 1587, 2197

Harris, M 859

Harris, SJ 2399

Hartmann, A 2164

Harvey, BJ 542

Harvey, R 1810

Harvey, RD 762

Hashida, Y 2624

Hashida, Y 629

Hassing, M-R 486

Hatae, M 613

Hata, H 170

Hatano, M 836

Hatiboglu, G 973

Hatlevoll, I 1712

Hawkins, MM 2455

Hayashi, Y 2123

Hayashi, Y 2516

Haybaeck, J 1830

Haydu, LE 924
Hayes, R 1378

Haynes, K 292

Haynes, R 775

Hazin, J 1449

Hazra, A 183

$\mathrm{He}, \mathrm{J} 1508$

Hei, TK 91, 1106

Heideman, DAM 1495

Heijnsdijk, EAM 1971

Heinemann, V 766, 469, 1684

Heinzerling, L 2164

Heinz, J 188

Heise, M 442

Heitz, D 1267

Hemon, D 1899

Henare, K 1306

Henderson, B 727

Henderson, S 964

Heng, DYC 2478

Heng, SJ 139

Henin, E 1810

Hennenlotter, J 131

Henson, KE 179

Hepworth, SJ 2178

Hernando, S 1732

Herr, I 1449

Herrera, F 2005

Herzig, K-H 1917

Hewish, M 983

Higashi, M 229

Higashi, S 2063

Hildenbrand, S 334

Hill, B 327

Hill, E 1602

Hiller, L 866

Hingorani, SR 1

Hinoda, Y 1659, 2070

Hirajima, S 361, 1324, 1822

Hirao, A 2516

Hirao, T 1119

Hiraoka, N 914, 951

Hirata, H 1659, 2070

Hiro, J 121

Hiroshima, K 744

Hirst, TC 64

Hiura, M 1957

Hiyoshi, H 2191

Ho, L 908

Hoang-Xuan, K 2178

Hochhauser, D 1252

Hochster, HS 477

Höckel, M 402

Hofbauer, F 1408

Hoff, PM 1316

Hofsli, E 1712

Hogervorst, FBL 2172

Hohenfellner, M 973

Hold, GL 2537

Holden, J 2186

Holdenrieder, S 1684

Holder, R 1149

Hollenbeck, AR 205, 1168

Hollingsworth, MA 2045

Holloway, R 1021

Holmberg, L 812

Holt, S 2250

Homann, N 519

Hong, D 826

Hong, YS 1862

Hoon, DSB 2033

Hooper, R 9

Hopmans, SN 2021

Hopper, JL 743

Hoppe-Seyler, F 973

Horgan, PG 139

Horn, L-C 402

Horn-Ross, PL 727

Hortobagyi, GN 285

Horwich, A 2399

Hoshino, I 644

Hoshino, S 653

Hosking, FJ 2178

Hoskin, PJ 2399
Houlston, RS 1502, 2178

Houssami, N 1579

Hovens, CM 1388

Howat, WJ 1340

Howie, CR 1883

Ho-Yen, C 2195

Høyer, S 420

Hsu, C 72

Hsu, L 285

Hu, C-y 1508

$\mathrm{Hu}, \mathrm{H}-\mathrm{M} 791$

$\mathrm{Hu}, \mathrm{N} 1378$

$\mathrm{Hu}, \mathrm{N} 784$

Huang, B 1998

Huang, H 380

Huang, X 503

Hughes, CM 1178

Hughes, TA 662

Huijgens, PC 2628

Huillard, O 1034

Huisman, C 881

Humphreys, I 2250

Hung, S-C 1778

Hunt, KK 1508

Hurst, CD 1368

Husicka, R 2448, 2470

Hutson, TE 311

Huttary, N 564, 570

Hutterer, GC 901

Huzarski, T 461, 2601

Hvidberg, L 292

Hwang, AE 1163

Iaffaioli, RV 1566

Iannacone, MR 240

Ichikawa, D 361, 1324, 1822

Idbaih, A 2178

Iddawela, M 866

Iftner, T 234

Ignatiadis, M 1358

Iida, K 836, 1415

Iijima, S 1119

Iizasa, T 744

Ikeda, M 2063

Ikeda, N 644

Ikoma, H 361

Illidge, T 2399

Imajoh, M 629, 2624

Imholz, ALT 2172

Im, S-A 1425, 1978

Inagaki, M 2063

Inam, I 1495

Inamine, A 1648

Ino, Y 914

Inoue, Y 121

Ionsecu, R 941

Iqbal, B 1552

Iqbal, ST 1552

Irving, G 2186

Irwin, M 602

Isaia, G 2525

Isbert, C 1432

Isella, C 2525

Ishii, N 2070

Islami, F 1391, 1552

Ismail, T 1149

Isozaki, Y 644

Itesako, T 1648

Ito, H 1817

Iwamoto, T 285

Iwamoto, Y 836

Iwasaki, M 914

Iwase, T 192

Izzo, F 222, 1566

Jaakkola, K 82

Jacobs, I 107, 2045

Jacobs, PA 959

Jäger, E 265, 519

Jäger, W 564, 570

Jahangir, A 2281

Jain, R 503

Jakesz, R 1408

Jakobsson, M 1541

Jakubowska, A 461, 2601

James, J 1149

James, L 2464

James, ND 1854

Jamieson, NB 997

Jang, SJ 1245

Jansen, L 699, 1579

Jansman, FGA 1071

Jardine, AKS 542

Jarzemski, P 461

Jassar, A 1288

Jayne, DG 662

Jayson, G 250

Je, Y 2478

Jefford, M 14

Jenkinson, C 1846

Jenkinson, HC 2455

Jenkins, RE 1846

Jenkins, V 1402, 2264

Jensen, KE 234

Jeong, BC 387

Jeong, S-Y 1425, 1978

Jeon, SS 387

Jersak, K 461

Jeziorski, K 301

Jia, J 2272

Jiang, W 380

Jiang, Z 859

Jiao, J 2542

Jiao, L 213

Jiménez-Hernández, E 2334

Jin, A 1182

Jirström, K 2321

Jithesh, PV 370

Jitsukawa, M 644

Jobsen, JJ 820

Johannesen, TB 1195

Johannesson, H 2321

Johansen, J 1712

Johansson, K-A 1964

Johansson, M 708

Johansson, SL 1079, 2590

Johnsen, JI 2191 
Kamieniak, MM 1732

Kamioka, M 629

Kamiura, S 1348

Kamura, S 836

Kamura, T 1957

Kanai, Y 914, 951

Kanekura, T 229

Kang, GH 1425, 1978

Kang, J-H 39

Kang, SN 39

Kang, W 2557

Kang, Y-K 1245

Kannappan, V 994

Kantola, T 1917

Kapoor, V 1288

Karadimou, A 887

Karamitopoulou, E 2088

Karapanagiotou-Schenkel, I 973

Karhu, T 1917

Karlsson, P 812

Karttunen, TJ 638, 1917

Kasamatsu, A 1817

Kasamatsu, T 1957

Kaseb, A 740

Kashimoto, K 1324

Kashyap, A 461, 442, 2601

Kato, H 2063

Kato, T 229

Katsumata, N 1957

Kauderer, J 613

Kauh, JS 762

Kauppila, JH 638

Kavallaris, M 2435

Kawabata, R 1119

Kawada, J 1119

Kawaguchi, T 361, 1324, 1822

Kawahata, S 1488

Kawakami, H 1488

Kawamoto, A 121

Kawamura, M 121

Kawano, Y 170

Kaye, SB 250, 1553

Kaymakcalan, MD 2478

Kazemier, HG 881

Keegan, TJ 1907

Kefford, RF 924

Keikavoussi, P 2164

Keller, S 1449

Keller, T 564

Kendall, GM 1907

Kennedy, SA 1061

Kermorgant, S 2195

Kern, C 1684

Kerr, M 748

Kerscher, AG 1432

Key, TJ 708

Khan, MS 1838

Khan, NA 229

Khawaja, S 2250

Khaw, K-T 708

Khoo, B 429

Khuri, FR 762

Khursheed, M 2056

Kiesewetter, F 2164

Kiesslich, T 1830

Kikkawa, N 1648

Kikuchi, E 2123, 2312

Kiltie, AE 748

Kim, BS 1245

Kim, D-W 39

Kim, GY 1245

Kim, J 39

Kim, JC 1862

Kim, K 387

Kim, KP 1862

Kim, S 1571

Kim, SY 1862

Kim, TW 1245, 1862

Kim, T-Y 1425, 1978

Kim, YS 1862

Kimura, T 1348

King, JC 1907

Kingston, JE 2455
Kinlen, LJ 1222

Kinoshita, T 1648

Kinsey, SE 1223

Kirchner, T 469

Kirita, T 1460

Kirkbride, P 9

Kirkwood, A 1252

Kiś, J 461

Kitteringham, NR 1846

Kjaer, SK 234

K Jagadeesan, S 721

Kjems, J 2291

Klæboe, L 2346

Klein, S 469

Klintrup, K 1917

Kluźniak, W 461, 2601

Knight, J 1340

Knowles, MA 1368

Knox, J 311

Koba, W 859

Kobayashi, H 1957

Kodama, S 1957

Koek, GH 676

Koelzer, VH 2088

Koenigsmann, M 265

Koepke, A 265

Koganti, S 2056

Kogevinas, M 742

Kogianni, G 163

Koh, W-P 1182

Kojouharoff, G 469

Kolla, JN 2056

Koller, K 1830

Kolonel, L 727

Komatsu, S 361, 644, 1324, 1822

Kondi-Pafiti, A 2088

Kong, L-L 1470

Konishi, H 361

Kop, JL 1012

Kopf, S 564, 570

Koriyama, C 229

Korkolopoulou, P 2142

Kornprat, P 1830

Kosaka, T 2123, 2312

Kosian, P 32

Koskas, M 1267

Kost, SE 155

Kosuge, T 914

Kotapalli, V 2056

Koutoulidis, V 2259

Kovacevic, Z 409

Kowalski, J 1541

Kratassiouk, G 2304

Kratz, C 1378

Kretschy, N 564, 570

Krieger, S 564, 570

Kristensen, H 420

Krivak, TC 1231

Krogh, V 708

Kroll, ME 2390

Krupitza, G 564, 570

Kruyt, FAE 587

Krzystolik, K 2601

Krzyzanowska, M 1298

Kubota, T 1822

Kuczyk, MA 131

Kudahetti, S 271

Kudo, T 1488

Kuehni, CE 2346

Kühnapfel, S 2164

Kullmann, F 265

Kume, M 629

Kuniyasu, H 1460

Kunzmann, V 1432

Kuppen, PJK 2033

Kurihara, M 1460

Kuroda, M 653

Kurokawa, Y 1119

Kurt, O 2625

Kurzrock, R 826

Kushima, R 951

Kusunoki, M 121

Kuteeva, E 2321
Kuwatani, M 1488

Kuzuya, K 1957

Kwasny, W 1408

Kwekkeboom, D 301

Labussière, M 2178

Lacey, JV 727

Lackner, C 1830

Ladekarl, M 2116

Lagerlund, M 292

Laghi, L 1750

Lagiou, A 199

Lagiou, P 199, 708

Lai, S-W 1778

Laios, I 1641

Lamar, M 21

Lambert, PC 691

Lambiase, A 58

Lambrechts, D 887, 1358

Lam, RKK 1106

Lam, WWT 1922

Lanchbury, JS 2582

Landi, L 668

Lane, B 370

Langdon, S 2039

Langley, RE 2192

Langridge, C 1402

Lannering, B 2346

Lara, PC 2565

Larne, O 1668

Larocca, LM 115

Larrañaga, N 708

Larsimont, D 1641

Larsson, A H 2321

Larsson, G 301

Lassa, A 2565

Lastoria, S 1566

Lathrop, M 2178

Lau, C 2178

Laubender, RP 469, 766

Launoy, G 775

Laurendeau, I 1720

Lauretti, GR 259

La Vecchia, C 222, 2354

Lawrence, G 1195

Lawson, MH 1340

Lazzeroni, M 1593

Le, NT 229

Leblanc, E 1267

Leblond, D 1012

Leclerc, J 2079

Lécuru, F 1267

Ledermann, JA 250, 2464

Lee, D-W 1978

Lee, HJ 1978

Lee, HM 387

Lee, J 39

Lee, JE 387

Lee, J-L 1245

Lee, KB 39

Lee, K-H 1425, 1978

Lee, M 1195

Lee, S-C 193

Leer, JWH 1784

Le Gouill, S 1801

Lehmann-Che, J 1807

Leithner, A 1677, 2627

Leitzmann, MF 798

Leja, M 941

Lejeune, S 2079

Leonardi, MC 1593

Lerut, E 887

Lesnock, JL 1231

Leung, HY 139, 149

Levidou, G 2142

Levy, L 1288

Lewandowski, PA 486

Lewis, CM 762, 2505

Lewis, IJ 1223

Lhotak, S 327

Li, B 2272

Li, C 2299

Li, F 1238

Li, M 2542

Li, P 1238

Li, Q 2381

Li, SA 848

Li, X 1508

Li, Y 99

$\mathrm{Li}, \mathrm{Z} 1870$

Liang, C-Z 1470

Liang, F 1870

Liang, Q 2557

Liang, X 2272

Liang, X 727

Liang, Y 380

Lianidou, ES 2426

Liao, B 1470

Liao, SY 613

Lichinitser, M 503

Liddle, S 908

Liegl-Atzwanger, B 1677

Lilja, H 1668

Liloglou, T 1332

Liloglou, T 370

Lim, T 1495

Linassier, C 887

Lin, C-Y 72

Lin, J 2372

Lin, K-L 72

Lin, S-R 791

Lin, T 380

Lin, W-H 1778

Lin, X 2470

Linch, DC 2399

Lindor, NM 1757

Ling, WHY 1931

Linseisen, J 708

Lips, EH 2172

Lissowska, J 727, 1378

Lister, TA 2399

Liu, H 941

Liu, JJ 183

Liu, P 1508

Liu, P 994 
2638

Luong, TV 1838

Luton, D 1267

Luu, HN 1173

Lyons, D 908

Lyratzopoulos, G 686, 1550, 2187

Ma, G 1238

Ma, H-H 1470

Ma, X-J 1332

Mabuchi, S 1348

Maccaroni, E 1126

MacCarthy, A 1907, 2455

Macher-Goeppinger, S 973

Mack, TM 1163

Macleod, MR 64

Macleod, U 681

Maeder, U 1432

Magliocco, A 727

Maher, J 14

Mahillo-Fernández, I 2565

Mahler-Araujo, B 602

Mahoney, DJ 245

Maïga, S 1801

Maisey, N 2505

Majumdar, D 1061

Mak, I 327

Makari, Y 1119

Makar, KW 1757

Mäkelä, J 1917

Makhdoomi, MA 1552

Mäkinen, MJ 1917

Makubate, B 1515

Mala, C 766

Malfertheiner, P 1750

Małkiewicz, B 461, 2601

Malmström, P-U 2321

Maloney, V 959

Maltby, P 1440

Mañas, A 2565

Mancuso, P 1387

Mandel, U 2045

Manikhas, G 503

Mann, KD 2339

Manning, JT 743

Mannweiler, S 901

Mansi, J 1936

Mansmann, U 469

Marangoni, E 1720

Marciano, R 1616

Marciniak, SJ 1340

Maretto, I 278

Marie, Y 2178

Marinaki, AM 2505

Maringe, C 1195

Marmorino, F 2549

Marmot, MG 2205

Marret, H 1267

Marsh, DJ 351

Marshall, H 1634

Marshall, MA 1998

Märten, A 469

Martin, A 1854

Martin, M 1027

Martin, N 429

Martin, RM 2329

Martin, SA 983

Martin, SD 155

Martín, V 2005

Martin, V 668

Martinez-Avalos, A 2334

Martínez-Javaloyas, JM 2565

Martín-Fernández, M 2565

Martini, M 115

Martinotti, M 1587

Martucci, R 1157

Marty, M 1807

Maruo, T 395

Maruyama, T 644

Masi, G 2549

Masojć, B 2601

Masood, A 1552

Massonnet, G 1720

Matczak, E 2470
Matei, D 1021

Mather, CB 1998

Mathevet, P 1267

Mathijssen, RHJ 1071

Matsubara, H 644, 951

Matsumoto, Y 1348

Matsumoto, Y 836

Matsuno, Y 1488

Matsuo, K 1348

Matsushima, S 1460

Matsushita, S 229

Matsuura, S 836

Mattick, JS 2419

Mattila, AE 638

Mattos, AL 259

Matych, J 461

Mauri, FA 621

Mauriz, JL 442

Mavroudis, D 2426

Mayer, A 1252

Mayer, A 402

Mayland, CR 1942

Mayne, ST 1168

Mayo, NE 1790

Mazzola, G 58

Mazzucchelli, L 668

McBurnie, J 1998

McCall, P 139

McCloud, RF 1949

McCormack, M 2464

McCormick, RI 1133

McCowan, C 1515

McCullough, ML 727

McDermott, DF 1393

McGahan, CE 1195

McGale, P 179

McGee, SL 1624

McKane, G 1440

McKay, CJ 997

McKinney, PA 1223, 1273

McLaren, CE 512

McLean, C 743

McLean, MH 2537

McMeekin, DS 1021

McMillan, DC 1915

McNally, RJQ 2339

McNeish, I 250

McPhail, S 686, 2187

Meckbach, D 2164

Medico, E 2525

Medina-Sansón, A 2334

Meech, SJ 1998

Meeran, K 429

Mehta, D 1061

Meijer, GA 1495

Méjean, A 887

Mejía-Aranguré, JM 2334

Mellor, D 771

Méndez, MJ 2565

Meng, F 579

Menkiszak, J 2601

Menon, U 107, 2045

Menzies, AM 924

Meredith, SL 1704

Merrick, H 1004

Merseburger, AS 131

Merson, S 2582

Mescoli, C 278

Meseguer, C 2565

Mesher, D 908

Meyer, T 995, 1252, 1838

Meyskens, FL 512

Mezzapelle, R 1743

Miccichè, F 1157

Michaelson, MD 1393

Michalski, CW 1846

Middleton, R 1195

Mier, JW 319

Miglio, U 1743

Mikami, S 2123, 2312

Miki, Y 1415

Mikulits, W 564

Mikulits, W 570
Milanesi, E 527

Milani, M 1587

Milenic, DE 2013

Miles, DW 1052

Miller, AB 542, 727

Miller, M 1273

Miller, RE 1553

Miller, S 2130

Millward, M 1998

Milne, K 155

Mimeault, M 1079

Minaki, P 199

Minami, T 1260

Minardi, D 1126

Min, YJ 1245

Mirabello, L 1378

Mir, O 1034

Mishalian, I 1288

Mistrangelo, DM 527

Mitchell, H 1810

Mitsunaga, S 2063

Mitsuya, H 170

Miwa, H 395

Miyagi, Y 1495

Miyajima, A 2123, 2312

Miyamoto, K-i 2516

Miyata, H 1119

Miyazaki, Y 2123

Mizunoe, T 1957

Mlineritsch, B 1408

Moebus, S 2178

Moe, M 2250

Mogushi, K 932

Mohri, Y 121

Moik, M 1408

Moldenhauer, G 1449

Mole, DR 1133

Molinari, F 1743

Molinari, F 668

Møller, H 149, 271, 691, 2582

Molnar, I 311

Monga, G 1743

Monson, K 2264

Montella, M 222

Montes, A 2264

Montgomery, N 542

Montironi, R 1126

Moore, K 351

Moore, LE 1378

Moore, MJ 1298

Moore, PS 2623

Moreau, P 1801

Morgan, DAL 1042

Morgan, L 1042

Morgan, SR 1316

Mori, M 644, 1119

Morimura, R 1822

Morimura, R 361

Morino, M 527

Mori, T 951

Morosky, A 1021

Morote, J 2565

Morris, E 775

Morris, EJA 681, 1502

Morris, K 1004

Morris, L 602

Morrow, CP 1348

Mortensen, NJ 2106

Morton, JP 997

Morvillo, M 2549

Moster, D 1525

Mottram, B 1624

Motzer, RJ 1571, 2470

Muenst, S 1480

Muir, K R 2601

Mulder, L 2172

Muller, DC 743

Müller, H 49

Müller-Nurasyid, M 2178

Müller, SC 973

Munoz, F 527

Muñoz-Repeto, I 1732

Munro, AJ 2433

Murias, A 2565

Murphy, F 2390

Murphy, MFG 1907, 2455

Murray, GI 2537

Murray, J 2435

Murray, LJ 1178

Murray, MJ 450

Muzzonigro, G 1126

Nabeshima, A 836

Nabi, S 1552

Nadella, P 762

Nagao, S 613

Nagata, H 2123

Nagata, H 361, 1324, 1822

Nagel, D 1684

Naimo, S 58

Naing, A 826

Najar, MS 1552

Nakada, M 2516

Nakagawa, K 2123

Nakagawa, M 1648

Nakajima, K 1119, 2070

Nakamura, Y 1119

Nakanishi, T 1957

Nakayama, E 1119

Naldi, N 1695

Nambu, E 2516

Namdarian, B 1388

Näpänkangas, J 1917

Nappi, L 1616

Narod, SA 461, 2601

Narvi, E 82

Nasti, G 1566

Nathwani, BN 1163

Neal, RD 25, 686, 721, 1550 ,

$$
2187
$$

Nedergaard, BS 2116

Nederlof, PM 2172

Neef, C 1071 
O'Beirne, J 1252

Öberg, A 2153

Obermann, EC 1480

Obi, N 188

O'Boyle, G 1634

O'Callaghan, CJ 784

O'Ceilleachair, A 1211

Ochiai, A 2063

Ochiai, T 361

O’Connell, MJ 784

Oda, Y 836

Odendaal, J 163

O’Donoghue, C 2200

Oertli, D 1480

Oetken-Lindholm, C 82

Ogawa, K 1348

Ogawa, R 951

Ogawara, K 1817

Ogino, S 1891

Oh, D-Y 1425, 1978

Ohi, M 121

Ohmori, H 1460

Ohno, I 2063

Ohno, S-i 653

Oh, ST 1245

Okabe, Y 395

Okada, Y 2123

Okada, Y 2312

Okamoto, K 361, 1324, 1822

Okamoto, Y 1648

Okazawa, M 1348

Okugawa, Y 121

Okuno, Y 170

Olde Damink, SWM 676

Olesen, F 1280

Oliver, S 681

Oliver, SE 1213

Olson, SH 727

Olsson, CE 1964

O'Mahony, JF 1209

O'Neill, A 319

O’Neill, KA 1907

Ono, M 836

Oonk, A 2172

Oosterwijk, JC 1579

Orihashi, K 629

Orlandi, A 115

Ormiston-Smith, N 2413

Ørntoft, TF 420

Osaka, Y 653

Osaki, Y 395

Osako, T 1921

Oshima, T 395

Osorio, A 1732

Osório, H 2045

Otsuji, E 361, 1324, 1822

Ottaiano, A 1566

Oudard, S 887

Ou, D-L 72

Ou, QJ 1238

Ou Shu, X 727

Overvad, K 708

Owonikoko, TK 762

Oya, M 2123, 2312

Pacey, A 1004

Pagani, G 1593

Paganotti, A 1743

Pagin, A 2079

Paish, EC 1042

Palacios, J 1732

Palaia, R 1566

Palazzo, F 429

Palli, D 708

Palmer, BD 1306

Palmer, DH 1440

Palmer, JR 727

Palmieri, C 163, 2039

Palmqvist, R 2153

Paludetti, G 1157

Pandey, P 2590

Pang, ASL 193

Pang, B 1495
Pan, Y-y 941

Papadimitriou, CA 1100

Papanikolaou, G 32

Papaspyrou, I 1100

Pappot, H 1994

Paradysz, A 461

Paraiso, D 1771

Paredes-Aguilera, R 2334

Paridaens, R 887

Park, B 387

Park, B-J 39

Park, BK 1245

Parker, C 420

Parker, CK 2367

Park, I 1245

Parkin, DM 1215

Park, J-G 1425

Park, J-G 1978

Park, JH 1425

Park, KJ 1425

Park, KJ 1978

Park, Y 1168

Park, Y 727

Park, YS 1245

Parry, MM 771

Parslow, RC 1223, 1273

Parveen, M 848

Pasquier, E 2435

Patanè, S 2525

Patard, J-J 887

Patch, D 1252

Patel, AV 727

Paterini, P 1750

Patsouris, E 2142

Patyna, S 1393

Pauligk, C 265, 519

Paulin, G 327

Pauwels, P 1358

Pavlakis, N 771

Pawlak, WZ 1994

Payne, S 1936

Pearce, MS 2339

Pedersen, AE 107

Pedersen, J 1388

Pedersen, JW 107, 2045

Peeters, DJE 1358

Peeters, M 503, 1358

Peggs, KS 1560

Pellat-Deceunynck, C 1801

Pelucchi, C 222

Pelz, JOW 1432

Peñaloza-Gonzalez, JG 2334

Penegar, S 1502

Pereira, SP 1113, 1252

Perne, C 1449

Perraki, M 549

Perren, T 250

Perrino, M 58

Perry, SL 662

Perumal, M 342

Pervin, S 848

Petersen, PM 1994

Peters, I 131

Peterson, JR 557

Peters, S 1879

Peters, TJ 25

Peto, J 1221

Petraki, K 2142

Petronis, J 1298

Pettersson, N 1964

Pett, MR 450

Peyromaure, M 1034

Pfeiffer, RM 1378

Pfeiler, G 1408

Phaeton, R 859

Phan, AT 826

Pharoah, PD 602

Phillips, CJ 2250

Phipps, AI 1757

Piazzi, G 1750

Piccirillo, MC 1566

Pichler, M 901, 1677, 1830, 2627

Picton, SV 1223
Pierga, J-Y 1807

Pietzner, K 32

Pinato, DJ 429, 621

Piñero-Madrona, A 1919

Pinna, G 2304

Pinthus, JH 327

Piotrowski, K 2601

Piperi, C 2142

Pirie, K 2390

Pischon, T 708

Pivot, X 1052

Plantade, A 1771

Platt, FM 1368

Ploner, F 1408

Plum, LM 301

Plummer, R 1634

Polesel, J 222

Pollack, JR 2056

Pollina, L 2549

Polo-García, L 1919

Pond, G 2021

Ponnusamy, MP 2590

Popovic, S 327

Pop, S 1027

Porchet, N 2079

Porter, DE 1883

Porto, E 2304

Poston, G 1440

Potter, JD 1757

Pouchy, C 2435

Poulin, EJ 1765

Poulsen, AH 2346

Prasnikar, N 265

Pratt, NR 873

Preisler, J 2448

Prescott, J 727

Presneau, N 964

Prestvik, WS 1712

Preusser, M 924

Pritchard, DM 1440

Prochazka, M 2346

Procopio, G 311

Provencher, L 1052

Provenzano, E 602, 866

Provenzano, PP 1

Pruneri, G 1593

Przybyła, J 2601

Przybyła, J 461

Psaltopoulou, T 2259

Pucciarelli, S 278

Pudney, D 2250

Pujade-Lauraine, E 1771

Pummer, K 901

Purdie, CA 873

Purshouse, K 163

Pusztai, L 285, 479

Puszyński, M 2601

Qian, W-C 1470

Qiao, Y-L 1378

Querleu, D 1267

Quezada, SA 1560

Quicios, C 2565

Quinlan, PR 873

Quintela-Fandino, M 1298

Quirke, P 681, 1502

Quispe-Tintaya, W 2281

Qureshi, U 1113

Raab, I 564, 570

Racca, P 527

Rachagani, S 2590

Rachet, B 775, 1195

Radford, JA 2399

Rae Bressler, L 1061

Rafiq, R 1552

Ragoussis, J 1133

Rajan, S 2442

Rajarajacholan, U 1143

Raj, KP 512

Ramachandran, R 429

Ramage, JK 301

Ramalingam, SS 762

Ramirez, AJ 292

Ramón y Cajal, T 1732

Rampazzo, E 278

Rangel-López, A 2334

Rao, H 380

Rashid, M 1113

Raslan, F 2448

Rassl, DM 1340

Rastogi, R 727

Ray-Coquard, I 1771

Recchia, F 1695

Redaniel, MT 2329

Reekie, A 1883

Reeves, G 2390

Reid, A 1879

Reis, CA 2045

Rena, O 1743

Renner, K 1408

Ren, Y 1508

Ress, AL 1830

Reyes-Zepeda, NC 2334

Reynes, G 2565

Rha, SY 39

Rhee, Y-Y 1978

Riabowol, K 1143

Ribal, MJ 2565

Riboli, E 708

Ricardi, U 527

Ricceri, F 708

Ricciardiello, L 1750

Richards, CJ 2478

Richards, JM 1061

Richards, MA 14, 1195, 1223

Richardson, DR 409

Richon, S 1720

Rico, D 1732

Ridaura, VK 1163

Rimassa, L 21

Rindi, G 1157

Ringberg, A 812

Rini, B 1571

Rini, BI 2470

Rintoul, RC 1340

Risch, H 1168 
Rose, JM 533

Rosenberg, L 727

Rose, PW 25, 721

Rosner, B 183

Rossetto, M 2178

Rossi, A 2415

Rössler, F 1100

Rossoni, G 58

Ross, P 2505

Rothé, F 1358

Rotkrua, P 932

Rotmensz, N 1593

Rots, MG 881

Roughton, M 1252

Roulson, J-A 1368

Rouzier, R 1267

Rouzier, R 285

Rowe, R 1004

Rowland, J 14

Różański, W 461

Rubin, GP 686, 1550, 2187

Rubio, G 2565

Rudant, J 1899

Rudraraju, B 2039

Ruiters, MHJ 881

Ruiz-Garcia, A 1393

Ruiz- Maciá, JA 1919

Rustgi, AK 755

Rutegård, J 2153

Rutherford, MJ 691

Rutten, A 1358

Ruud, E 1525

Ryan, DP 1393

Rye, M 1712

Ryu, M-H 1245

Saetta, A 2142

Sáez, R 1732

Saigusa, S 121

Saiko, P 570

Saini, HK 450

Saito, K 1817

Saito, T 1957

Sai, Y 2516

Sakalidou, M 2142

Sakamoto, N 1488

Sakurai, M 1415

Salamanca-Gómez, F 2334

Salès, F 1641

Sales, MJ 873

Saletti, P 668

Sali, M 1157

Salnikov, AV 1449

Salo, T 638

Salto-Tellez, M 741

Salvatore, L 2549

Samaras, V 2142

Samoli, E 199

Samonigg, H 1408, 1677, 1830

Samper, P 2565

Sampson, J 1168

Sanchez-Carbayo, M 1368

Sánchez, E 2565

Sánchez, M-J 708

Sanchez-Sanchez, AM 2005

Sanchez, WY 1624

Sandbichler, P 1408

Sandelin, K 812

Sandell, S 438

Sanderson, JD 2505

Sandin, R 1541

Sandström, P 1541

Sangale, Z 2582

Sangi-Haghpeykar, H 213

Sanguinetti, M 1157

San José, LA 2565

Sanli, T 2021

Sansom, OJ 997

Sanson, M 2178

Santangelo, R 1157

Santini, S 2495

Santoro, A 21, 58

Sargent, DJ 784
Sarlak, H 2625

Sasahira, T 1460

Sasano, H 1415

Sasieni, PD 1215

Sastry, RA 2056

Sathyanarayana, UG 744

Sato, H 2516

Sato, Y 2033, 2123

Savage, PM 1810

Savage, RE 21

Savage, SA 1378

Savvatis, K 32

Sayasneh, A 2448

Scardino, P 149, 271, 2582

Scarpini, CG 450

Scartozzi, M 1126

Schache, AG 1332

Schache, AG 370

Schaeffer, C 1061

Schairer, C 727

Schauer, S 1830

Scherer, R 131

Scherer, SJ 1052

Scheurer, ME 1173

Schinzari, G 115

Schirripa, M 2549

Schlange, T 1449

Schlischewsky, N 402

Schmalenberg, H 519

Schmidberger, H 402

Schmidt, LS 2346

Schmidt, S-C 32

Schmiedel, S 234

Schmiegelow, K 2346

Schmoll, H-J 1316

Schneider-Stock, R 2164

Schober, T 49

Schoedel, J 1133

Schoemaker, M 2178

Schöffski, P 887

Scholefield, J 2097

Schouten, LJ 727

Schramm, J 2178

Schreiber, S 2178

Schröder, FH 1971

Schrøder, H 1280

Schrøder, HD 715

Schuit, RJL 438

Schuler, G 2164

Schüz, J 2346

Schwartz, B 21

Schwartz, PE 1021

Schwarzberg, AB 1393

Schwientek, T 2045

Scolyer, RA 924

Scorilas, A 2573

Scott, V 1807

Scowcroft, H 2413

Seckl, MJ 1810

Segarra, J 2565

Segersten, U 2321

Sehouli, J 32

Seibold, P 188

Seifert, M 1408

Sekikawa, A 395

Seki, N 1648

Sekine, S 951

Selby, PJ 1133

Selgrad, M 1750

Selim, U 1408

Seminog, OO 193

Sena, ES 64

Senetta, R 527

Senzer, N 21

Seo, SI 387

Sergentanis, TN 2259

Sermage-Faure, C 1899

Serrano, D 1593

Serth, J 131

Seshacharyulu, P 2590

Sessa, C 1021

Setiawan, VW 727

Setsu, N 836
Severi, G 743

Severo, M 2354

Seywright, M 139

Sfikas, C 199

Shafi, R 1552

Shah, IA 1552

Shahryari, V 1659

Shah, SA 1552

Shah, SIA 2192

Shaked, Y 1387

Shamonki, JM 2033

Shapiro, GI 1393

Shapiro, J 503

Sharaiha, Y 2250

Sharma, NL 748

Sharma, R 429, 621

Sharp, L 1211

Shaw, RJ 370, 1332

Shaw, V 1846

Shemais, T 964

Sheng, H 1870

Shenton, G 1223

Shen, X 1870

Shen, Y-C 72

Shete, S 2178

Shibata, K 1119

Shibata, T 1957

Shibli, S 351

Shigematsu, H 744

Shi, H 2542

Shiiba, M 1817

Shilkaitis, A 2495

Shimada, K 914

Shimada, S 932

Shimizu, S 2063

Shimwell, NJ 1854

Shin, DM 762

Shinozuka, K 1817

Shiozaki, A 361, 1324, 1822

Shi, Q 784

Shivapurkar, N 744

Shuda, M 2623

Siesling, S 1579

Sieuwerts, AM 1358

Signoretti, S 319

Sigurdson, AJ 1378

Sikorska-Radek, P 461

Sikorski, A 461, 2601

Silva, L 2045

Simon, AE 292

Simonds, H 2464

Simonelli, M 21, 58

Simon, M 2178

Singer, C 1408

Singhal, S 1288

Singh, G 2021

Singh, LB 149

Singh, R 848

Sinha, R 205

Sita-Lumsden, A 1925

Siu, LL 1298

Sjursen, W 1712

Skrzypczyk, M 461

Sloan, P 1332

Słojewski, M 2601

Słupski, P 461

Smith, AA 2448

Snoek, FJ 2628

Soczawa, M 2601

Soe, PP 1931

Sola-Pérez, J 1919

Solis-Trapala, I 2264

Solsona, E 2565

Song, M 189

Song, Q 941

Song, T 1870

Song, Y 1508

Sonpavde, G 2478

Sorensen, B S 2291

Sørensen, HT 1189

Sørensen, KD 420

Sorensen, S 2291

Soria, D 1042

Sosnowski, M 461, 2601

Soysal, SD 1480

Spanos, E 199

Sparber, C 2259

Spatz, A 1641

Speirs, R 1388

Spitale, A 668

Spizzo, G 1480

Spyratos, F 1807

Srivastava, S 741

Staines, A 1211

Stalder, C 2448

Stalmeier, PFM 1784

Stamp-Vincent, C 2039

Stanbridge, EJ 613

Stapley, S 25

Stark, D 1004, 1223

Stary, S 564

Stathis, A 1298

Stattin, P 708

Steele, CW 997

Stefano, ALD 2178

Steger, GG 1408, 2259

Steineck, G 1964

Stein, J 1092

Steinmetz, C 442

Stenzl, A 131

Stephenson, P 1393

Steptoe, A 2407

Stevens, AM 2399

Stevens, MCG 2329

Steward, J 1195

Steyerberg, EW 533

Stieber, P 1684

Stiller, CA 2455

Stockton, DL 1883

Stoeckigt, C 901

Stöger, H 1408

Stojakovic, T 901, 1677, 1830

Stojdl, DF 245

Stolzenberg-Solomon, RZ 1168

Stolzenburg, S 881 
Szekeres, T 564

Szekeres, T 570

Szkandera, J 1677, 2627

Taal, B 301

Tabár, L 812

Tabatabai, ZL 1659, 2070

Tabernero, AJ 2565

Taboada, M 1316

Taghipour, S 542

Taguchi, T 629

Takahashi, T 1119

Takahashi, T 744

Takanashi, M 653

Takasawa, A 1488

Takeshita, H 361, 1324, 1822

Takeshita, N 644

Takiguchi, S 1119

Takiguchi, Y 1817

Takino, T 2516

Talamini, R 222

Tanaka, H 932

Tanaka, K 121

Tanaka, N 2123

Tanaka, Y 1659

Tan, DSP 1553

Tan, IB 1495

Taniguchi, A 629

Taniguchi, H 361, 951

Tannahill, C 139

Tan, P 1495

Tan, TM 429

Tanzawa, $\mathrm{H} 1817$

Tappenden, P 1211

Tarazi, J 1571

Taylor, C 179

Taylor, CF 1368

Taylor-Papadimitriou, J 2045

Taylor, PR 1378

Tebbutt, NC 503, 771

Teichmann, M 564, 570

Teixeira, AS 1113

Tejpar, S 668

Tembo, O 1807

Terao, K 2063

ter Haar, NT 873

Terry, G 908

Teucher, B 708

Tezval, H 131

Thalappilly, S 1143

Themistocleous, MS 2142

Thielen, I 49

Thomale, J 334

Thomas, AL 2417

Thomas, F 1534

Thomas, JD 681

Thommesen, L 1712

Thompson, AM 873, 1515

Thompson, JF 924

Thompson, SG 2205

Thomson, AJ 2537

Thomson, CS 2413

Thrall, MM 1231

Tian, C 1231

Tiernan, JP 662

Tighiouart, M 762

Tijono, SM 1306

Tikishvili, E 2582

Tilson, L 1211

Timmer-Bosscha, H 587

Timmerman, D 2448

Timofeeva, I 58

Tirabosco, R 964

Tisch, U 941

Tiseo, M 1695

Tishelman, C 292

Tjønneland, A 708

Tlemsani, C 1034

Tod, M 1810

ToftSørensen, H 715

Toganel, C 1027

Toiyama, Y 12

To, K-F 2557
Tomata, Y 2381

Tomczak, P 1052

Tomita, T 395

Tommasino, M 240

Tonack, S 1846

Tong, JH-M 2557

Tong, Z-T 1470

Toro, J 1378

Torres-Nava, JR 2334

Tortora, G 1616

Toumpanakis, C 1838

Tourani, J-M 887

Toussi, STK 429

Tracey, E 1195

Trahair, T 2435

Tran, L 848

Tran $\varnothing$, G 1712

Tran, UM 1143

Trichopoulos, D 199, 708

Trichopoulou, A 708

Trinder, P 2045

Troncone, G 1616

Trousil, S 342

Tsai, H-L 791

Tsai, P-Y 1778

Tsakiridis, T 2021

Tsiani, E 2021

Tsigginou, A 1100

Tsikkinis, A 199

Tsochatzis, E 1252

Tsuburaya, A 1495

Tsuchida, A 653

Tsuda, H 951, 1324

Tsuji, I 2381

Tsuji, T 2516

Tsujiura, M 361, 1822

Tsumura, T 395

Tsuta, K 951

Tsutsui, T 1348

Tucker, MA 1378

Tucker, SL 1964

Tumino, R 708

Tuomisto, A 1917

Turati, F 222

Turner, D 1195

Turner, RR 2033

Tye, L 1393

Tynes, T 2346

Uchida, K 121

Uemura, H 1260

Ueno, K 1659

Ueno, K 2070

Uen, Y-H 791

Uhlén, M 2321

Uhlén, P 2191

Ulhøi, BP 420

Ulmert, D 708

Unda, M 2565

Underhill, C 1998

Underwood, MA 139

Uppin, SG 2056

Urioste, M 1732

Urman, R 848

Urup, T 1994

Usher, C 1211

Uzawa, K 1817

Vacher, S 1720

Vaira, V 621

Valencia, T 597

Valentine, W 2250

Valentini, V 1157

Validire, P 1720

Vallier, A-L 866

Van Cutsem, E 493

van Dam, PA 1358

van den Brandt, PA 727

van den Broek, MAJ 676

Van den Eynden, GG 1358

van der Gun, BTF 881

van der Kolk, LE 2172

van der Linden, MHM 2628 van der Palen, J 820

van de Velde, CJH 2033

van de Wiel, MA 1732

VanDongen, S 450

van Gelder, T 1071

van Grieken, NCT 1495

Van Hazel, GA 771

van Hoesel, AQ 2033

Van Kempen, LC 1641

van Kranen, HJ 708

van Laar, M 1223, 1273

Van Laere, SJ 1358

van Leeuwen, RWF 1071

van Lin, EJNT 1784

van Meijel, B 2628

van Oort, IM 1784

van Strijp, JAG 587

van Tol-Geerdink, JJ 1784

Vardakis, N 549

Varricchio, C 1593

Vasile, E 2549

Vassilarou, D 199

Vaughan, VC 486

Vaupel, P 402

Väyrynen, JP 1917

Vázquez, F 2565

Vedsted, P 292, 1280

Vehling-Kaiser, U 469

Velazquez-Aviña, MM 2334

Vellone, VG 1157

Veneziani, BM 1616

Verghese, ET 662

Vergine, M 429

Vergunst, H 1784

Verheul, HM 2628

Verkarre, V 887

Vermeulen, KM 1579

Vermeulen, PB 1358

Vesterinen, HM 64

Viale, G 1593

Viehl, CT 1480

Vigmostad, MN 292

Vignat, J 240

Villano, JL 2367

Vincent, TJ 1907, 2455

Vinjamuri, S 1440

Viola, K 564

Viola, K 570

Virizuela, JA 2565

Virkus, RA 727

Vislovukh, A 2304

Visser, O 2628

Viswanath, K 1949

Vonach, C 564

Vonach, C 570

von Deimling, A 924

Vora, J 1440

Vornanen, J 1917

Vo Van, M-L 1985

Vrettakos, G 2142

Vreuls, CPH 676

Vrieling, A 188

Vuckovic, S 162

Vyas, M 2097

Wacrenier, A 2079

Wada, H 1119

Wagener, N 973

Wagstaff, J 1402

Wahlgren, T 1541

Wakasa, T 395

Wakelam, MJ 1149

Walenkamp, AME 587

Walker, JL 613

Walland, K 866

Waller, A 738, 2631

Wallis, MG 2442

Walsh, C 1211

Walter, FM 25

Walter, H 2417

Walters, S 1195

Walton, NA 602

Wandall, HH 107, 2045

Wang, H 1332

Wang, J 2372

Wang, J-Y 79

Wang, L 1298

Wang, L-CS 1288, 1306

Wang, LM 2106

Wang, R 1182

Wang, W 994

Wang, X 319

Wang, XG 859

Wang, Y 1765

Wang, Y 1936

Wang, Y 2542

Warburton, F 292

Ward, DG 1854

Ward, DM 450

Ward, JH 2130

Ward, LC 1495

Wardle, J 292, 831, 2407

Ward, MH 205

Ward, ST 1149

Wareham, NJ 708

Warmann, SW 334

Warmington, S 1149

Wärnberg, F 1195

Warner, D 503

Warren, AY 597

Warso, MA 106

Warwick, V 2464

Wasmuth, HH 1712

Wason, J 1340

Watanabe, T 2381

Watari, J 395

Watkins, J 1838

Watson, E 1602

Watson, PH 155

Waxman, J 163, 1925

Weber, B 1771

Webster, S 1298

Weijerman, PC 1784

Wei, J-H 1470

Weissman, H 1440

Weiswald, L-B 1720

Wei, W 1854

Wei, Y 1508 
Win, AK 1757

Winkelmann, C 469

Winsel, S 82

Winstanley, A 676

Wise, LA 727

Wisse, E 676

Witjes, JA 1784

Wittwer, C 1684

Wojnar, L 461

Wokołorczyk, D 461, 2601

Wolter, P 887

Wong, CKE 1388

Wong, GS 755

Wong, RPW 493

Wong, YNS 812

Wood, CG 319

Woodley, L 163

Wright, C 908

Wright, J 2021

Wright, M 1634

Wright, NA 741

$\mathrm{Wu}, \mathrm{B} 503$

$\mathrm{Wu}, \mathrm{H}-\mathrm{C} 1778$

Wu, J 99

Wu, K 1891

$\mathrm{Wu}, \mathrm{W} 653$

$\mathrm{Wu}, \mathrm{X} 2372$

Wu, Y-Y 2241

Wynants, L 2448

Xenidis, N 549

Xiang, Y-B 727

Xie, D 1470

Xing, X 2542

Xiong, F-x 941
Xu, B 1985

Xu, D 2272

Xue, Z 1870

$\mathrm{Xu}, \mathrm{J} 1508$

$\mathrm{Xu}, \mathrm{W}-\mathrm{H} 727$

$\mathrm{Xu}, \mathrm{Z}$-q 941

Xyrafas, A 549

Yadegarfar, G 301

Yaegashi, N 1957

Yamada, H 836

Yamada, T 1061, 2495

Yamamoto, K 1460

Yamasaki, M 1119

Yamazaki-Itoh, R 914

Yang, DH 1245

Yang, H 1378

Yang, HP 727

Yang, J 1508

Yang, XR 1378

Yang, ZH 149, 271, 2582

Yaniv, I 1092

Yan, Y 2590

Yao, M 409

Yao, Z 2299

Yasugi, T 1957

Yeager, M 1378

Yeluri, S 662

Yen, M-F 2241

Yeung, TM 2106

Yi, M 1508

Ylstra, B 1732

Yokota, H 1957

Yoneyama, Y 644

Yong, KJ 2013
Yook, JH 1245

Yoon, YS 1862

Yoshida, Y 2516

Yoshikawa, H 1957

Yoshikawa, T 1495

Yoshimine, S 2312

Yoshimura, K 1260

Yoshino, H 1648

Yothers, GA 784

You, B 1810

Young, A 1298

Young, AC 1133

Yuan, J-M 1182

Yuasa, Y 932

Yu, C-P 224

Yu, D 1252

Yu, G 1163

Yu, J 2557

Yuki, H 170

Yu, MC 1182

Yu, Y 579

Zacherl, M 1677

Zagonel, V 278

Zagouri, F 1100, 2259

Zahn, C 123

Zaikin, A 107

Zanelli, F 1695

Zang, DY 1245

Zannino, D 771

Zannoni, GF 1157

Zanoni, V 1587

Zaragoza-Zaragoza, C 1919

Zargar, SA 1552

Zattoni, F 2415
Zdrojowy, R 461, 2601

Zeegers, MP 1854

Zelenika, D 2178

Zell, JA 512

Zerbib, M 1034

Zerimech, F 2079

Zhang, D 409

Zhang, F 1470

Zhang, J -X 1470

Zhang, L 319

Zhang, N 99

Zhang, SL 1495

Zhang, W 99

Zhang, X 395

Zhang, YE 924

Zhang, Z 1870

Zhao, I 2557

Zhao, Y 1870

Zhao, Y 1985

Zhong, L 738

Zhong, M 579

Zhou, H 1106

Zhou, H 91

Zhu, AX 1393

Ziegler, RG 1378

Zietman, AL 1225

Zigeuner, R 901

Zisakis, A 2142

Zlobec, I 2088

Zografos, GC 2259

Zoumas-Morse, C 512

Zourna, P 199

Zucali, P 21

Zucali, PA 58

Zucman-Rossi, J 887 